International Journal of Pure and Applied Mathematics

Volume 104 No. 3 2015, 285-298

ISSN: 1311-8080 (printed version); ISSN: 1314-3395 (on-line version)

url: http://www.ijpam.eu

doi: http://dx.doi.org/10.12732/ijpam.v104i3.1

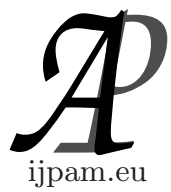

\title{
FREE CONVECTION BOUNDARY LAYER FLOW OVER A STRETCHING FLAT PLATE WITH VARIABLE THERMAL CONDUCTIVITY AND RADIATION
}

\author{
M.Y. Malik ${ }^{1}$, S. Bilal ${ }^{2}$, Farzana Khan $^{3 \S}$ \\ $1,2,3$ Department of Mathematics \\ Quaid-E-Azam University \\ 45320, Islamabad, 44000, PAKISTAN
}

\begin{abstract}
An analytical approach for the study of effect of thermal radiation on steady boundary layer flow with variable thermal conductivity due stretching sheet has been developed. To describe radiative heat flux, Rosseland diffusion approximation energy equation is used. The governing equation reduced to similarity boundary equation using suitable transformation and then solved by using homotopy analysis method. The velocity profiles and temperature profiles are compared numerically by using Fehlburg method.
\end{abstract}

Key Words: free convection, stretching plate, radiation, variable thermal conductivity, skin-friction coefficient, and Nusselt number

\section{Introduction}

The common type of body force which acts on a fluid, is due to gravity. The study of fluid flow gained significant importance in engineering disciplines. For example free convection flow over vertical flat plate has attracted researchers because of its application in cooling by natural convection as in case of electrical heaters and transformer. Also the study of natural convection has supported

Received: September 23, 2013

(C) 2015 Academic Publications, Ltd.

$\S_{\text {Correspondence author }}$ url: www.acadpubl.eu 
scientists when stars and planets or cooling reactors is required.

In a manufacturing process of a flat plate, application of boundary layer flow demands special attention. Example of such technological process of hot rolling, wire drawing, glass fibre and paper production. The moving fiber is governed by rate at which it is cooled. A number of work are presently available that follows the pioneer work of Crane [1], Angew, Chen and char [2], Datta, Roy, Gupta [3]. In 2010 Raftari, Yildrim [5] considered heat transfer problem with variable thermal conductivity on flow towards stretching sheet, Ariel [6] also studied computation of flow past a stretching sheet. In most of cases thermal conductivity is considered to be constant But in this paper we try to solve the boundary layer flow of viscous incompressible flow past a flat plate.

Many transport processes that occur both in nature and in industries involve fluid flows with combined heat and mass transfer. Such flows are driven by the buoyancy effects arising from the density variations caused by the variations in temperature and/or species concentrations

In some industrial problems dealing with chemical reactions and dissociating fluids, heat generation/absorption and chemical effects are very important. Vajravelu, Hadjinicolaou[12]. Some of the work related to the paper can be found in the papers by Mukhopadhayay [13], Ali [14], Ishak, Nazar and Pop [15] and chiam [16].

\section{Mathematical Formulation}

Consider the steady, incompressible and two-dimensional boundary layer free convection flow of a viscous fluid over a flat plate with variable thermal conductivity and radiation effects. Here we assumed that thermal conductivity varies linearly with temperature. The governing boundary equations are

$$
\begin{gathered}
\frac{\partial(u)}{\partial x}+\frac{\partial(v)}{\partial y}=0, \\
u \frac{\partial u}{\partial x}+v \frac{\partial u}{\partial y}=\nu \frac{\partial^{2} u}{\partial y^{2}}+g \beta\left(T-T_{\infty}\right), \\
\bar{u} \frac{\partial v}{\partial \bar{x}}+\bar{v} \frac{\partial v}{\partial \bar{y}}=\nu \frac{\partial^{2} v}{\partial \bar{y}^{2}}+\frac{\partial^{2} u}{\partial \bar{y}^{2}}, \\
u \frac{\partial T}{\partial \bar{x}}+v \frac{\partial T}{\partial \bar{y}}=\frac{1}{\rho c_{p}} \frac{\partial}{\partial y}\left(\alpha \frac{\partial T}{\partial y}\right)-\frac{1}{\rho c_{p}} \frac{\partial q_{r}}{\partial r},
\end{gathered}
$$


subjected to the boundary conditions:

$$
\begin{aligned}
& u=0, \quad v=v_{w}(x), \quad T=T_{\infty} \quad \text { at } x=0, \\
& u=0, \quad v=0, \quad T=T_{w}(x) \quad \text { as } y \rightarrow \infty .
\end{aligned}
$$

In the above expressions, $u$ and $v$ are the velocity components in $x$ and $y$ directions respectively, $q_{r}$ is the radiative heat flux, $\nu$ is the kinematic viscosity, $\rho$ is the density, $c_{p}$ is the specific heat, $\alpha(T)$ is the variable thermal conductivity, $T$ and $T_{\infty}$ are the temperatures of the fluid and surrounding respectively,.

There are few forms of thermal conductivity variation available in literature Among them we have considered that one which is appropriate for liquid introduced by Hossain et [16] as follows

$$
\alpha=\alpha_{\infty}\left(1+\in\left(T-T_{\infty}\right)\right),
$$

where $\alpha_{\infty}$ is the thermal conductivity of ambient fluid and

$$
\in=\frac{1}{\alpha_{f}}\left(\frac{\partial \alpha}{\partial T}\right)
$$

Using Roseland approximation the radiative heat flux is simplified as:

$$
q_{r}=-\frac{4 \sigma^{*}}{3 k^{*}} \frac{\partial T^{4}}{\partial y}
$$

where $\sigma^{*}$ and $\mathrm{k}^{*}$ are the Stefan-Boltzmann constant and the mean absorption coefficient, We assume that the temperature differences within the flow,are assumed to be sufficiently small such as the term T4, may be expressed as a linear function of temperature. Hence, this is accomplished by expanding T4 in a Taylor series about $\mathrm{T} \infty$ and neglecting higher-order terms, we get

$$
T^{4}=T_{\infty}^{4}+\left(T-T_{\infty}\right) 4 T_{\infty}^{3} .
$$

Using the similarity transformations

$$
\begin{aligned}
\Psi & =x^{\frac{3}{4}}\left(T_{w(x)}\right)^{\frac{1}{4}} f(x, \eta), \quad T=T_{w}(x) \Theta(x, \eta), \\
\eta & =T_{w}(x) \frac{y}{x^{\frac{1}{4}}}
\end{aligned}
$$

Equation (1) is automatically satisfied while Eqs. (2) to (4) takes the form

$$
f_{\eta \eta \eta+} \frac{(3+M)}{4} f f_{\eta \eta}-\frac{1}{2}(1+M) f^{\prime 2}+\theta=0,
$$




$$
\begin{gathered}
\left.\frac{1}{\operatorname{Pr}}\left((1+\epsilon+\epsilon \theta) \theta^{\prime \prime}+\frac{4 R}{3}\right) \theta^{\prime \prime}\right)+\frac{3+M}{4} f \theta_{\eta}-M f_{\eta} \theta=0 \\
f(0)=0, \quad f^{\prime}(0)=1, \quad \theta(0)=1 \\
f^{\prime}(\infty)=0, \quad \theta(\infty)=0 .
\end{gathered}
$$

Where $M, \operatorname{Pr}, R$, and $\in$. $M$ denotes the Temperature parameter, $P r$ denotes Prandtl number $R$ is representing radiation parameter and $\in$ denotes variable thermal conductivity parameter which are given by

$$
\begin{aligned}
& \operatorname{Pr}=\frac{\mu c_{p}}{\alpha_{\infty}}, \\
& M=\frac{x}{T_{w}(x)} \frac{d T_{w}}{d x}
\end{aligned}
$$

\section{Homotopy Solutions}

Homotopy analysis method depend upon the initial guesses $\left(f_{0}, \theta_{0}\right)$ and linear operators $\left(\mathcal{L}_{f}, \mathcal{L}_{\theta}\right)$ which are given in the forms

$$
\begin{gathered}
f_{0}(\eta)=0, \quad \theta_{0}(\eta)=\exp (-\eta), \\
\mathcal{L}_{f}(\eta)=\frac{d^{3} f}{d \eta^{3}}-\frac{d f}{d \eta}, \quad \mathcal{L}_{\theta}(\eta)=\frac{d^{2} \theta}{d \eta^{2}}-\theta,
\end{gathered}
$$

with

$$
\begin{gathered}
\mathcal{L}_{f}[C 3 * \exp (-\eta)+C 1+C 2 * \eta]=0, \\
\mathcal{L}_{\theta}[C 1 * \exp (-\eta)]=0=0,
\end{gathered}
$$

where $A_{i}(i=1-3)$ are the arbitrary constants. The zeroth and $m$ th order deformation problems are 


\subsection{Zeroth-Order Problem}

$$
\begin{gathered}
(1-p) \mathcal{L}_{f}\left[\hat{f}(\eta ; p)-\hat{f}_{0}(\eta)\right]=p \hbar_{f} N_{f}[\hat{f}(\eta ; p), \hat{\theta}(\eta ; p)] \\
(1-p) \mathcal{L}_{\theta}\left[\hat{\theta}(\eta ; p)-\hat{\theta}_{0}(\eta)\right]=p \hbar_{\theta} N_{\theta}[\hat{f}(\eta ; p), \hat{\theta}(\eta ; p)] \\
\widehat{f}(0 ; p)=0, \quad \widehat{f}(0 ; p)=1, \quad \widehat{f}(\infty ; p)=0 \\
\widehat{\theta}(0 ; p)=1, \quad \widehat{\theta}(\infty ; p)=0 \\
N_{f}[\hat{f}(\eta ; p), \hat{\theta}(\eta ; p)]=\frac{\partial^{3} \hat{f}(\eta ; p)}{\partial \eta^{3}}+\left(\frac{3+M}{4}\right) \hat{f}(\eta ; p) \frac{\partial^{2} \hat{f}(\eta ; p)}{\partial \eta^{2}} \\
\left.\mathcal{N}_{\theta}[\widehat{\theta}(\eta ; p), \widehat{f}(\eta ; p)]=\frac{1}{\operatorname{Pr}}(1+\epsilon \widehat{\theta}(\eta, p)) \frac{\partial^{2} \widehat{\theta}(\eta, p)}{\partial \eta^{2}}+\frac{1}{\operatorname{Pr}}\left(1+\frac{4 R}{3}\right) \frac{\partial \hat{f}(\eta ; p)}{\partial \eta}\right)^{2}+\theta \\
-\left(\left(\frac{3+M}{4}\right) \hat{f}(\eta ; p) \frac{\partial \hat{\theta}(\eta ; p)}{\partial \eta}+(M) \frac{\partial \hat{f}(\eta ; p)}{\partial \eta} \hat{\theta}(\eta ; p)\right)
\end{gathered}
$$

where $p \in[0,1]$ is embedding parameter and $\hbar_{f}$ and $\hbar_{\theta}$ are the non-zero auxiliary parameters.

\section{2. $m$-th-Order Deformation Problems}

$$
\begin{gathered}
\mathcal{L}_{f}\left[f_{m}(\eta)-\chi_{m} f_{m-1}(\eta)\right]=\hbar_{f} \mathcal{R}_{m}^{f}(\eta), \\
\mathcal{L}_{\theta}\left[\theta_{m}(\eta)-\chi_{m} \theta_{m-1}(\eta)\right]=\hbar_{\theta} \mathcal{R}_{m}^{\theta}(\eta), \\
f_{m}(0)=f_{m}^{\prime}(0)=f_{m}^{\prime}(\infty)=0,
\end{gathered}
$$




$$
\begin{gathered}
\theta_{m}(0)=1 \quad \theta_{m}(\infty)=0, \\
\mathcal{R}_{m}^{f}(\eta)=f_{m-1}^{\prime \prime \prime} \\
+\left(\frac{3+M}{4}\right) \sum_{k=0}^{m-1} f_{k} f_{m-1-k}^{\prime \prime}+\left(\frac{1+M}{2}\right) \sum_{k=0}^{m-1} f_{k}^{\prime} f_{m-1-k}^{\prime}+\theta_{m-1} \quad(32) \\
R_{m}^{\theta}(\eta)=\frac{1}{\operatorname{Pr}}\left(1+\frac{4 R}{3}\right) \theta_{m-1}^{\prime \prime}+\frac{1}{\operatorname{Pr}}\left(1+\in \theta_{m-1}\right) \theta_{m-1}^{\prime \prime}+\left(\frac{3+M}{4}\right) \sum_{k=0}^{m-1} f_{k} \theta_{m-1-k}^{\prime} \\
\chi_{m}=\left\{\begin{array}{c}
m-1 \\
0, \quad m \leq 1 \\
1, \quad m>1
\end{array} .\right.
\end{gathered}
$$

For $p=0$ and $p=1$, we can write

$$
\begin{gathered}
\widehat{f}(\eta ; 0)=f_{0}(\eta), \quad \widehat{f}(\eta ; 1)=f(\eta), \\
\widehat{\theta}(\eta ; 0)=\theta_{0}(\eta), \quad \widehat{\theta}(\eta ; 1)=\theta(\eta)
\end{gathered}
$$

and with the variation of $p$ from 0 to $1, \widehat{f}(\eta ; p)$ and $\widehat{\theta}(\eta ; p)$ vary from the initial solutions $f_{0}(\eta)$ and $\theta_{0}(\eta)$ to final solutions $f(\eta)$ and $\theta(\eta)$ respectively. By Taylor's series we have

$$
\begin{array}{cc}
\widehat{f}(\eta ; p)=f_{0}(\eta)+\sum_{m=1}^{\infty} f_{m}(\eta) p^{m}, \quad f_{m}(\eta)=\left.\frac{1}{m !} \frac{\partial^{m} \widehat{f}(\eta ; p)}{\partial p^{m}}\right|_{p=0} \\
\widehat{\theta}(\eta ; p)=\theta_{0}(\eta)+\sum_{m=1}^{\infty} \theta_{m}(\eta) p^{m}, \quad \theta_{m}(\eta)=\left.\frac{1}{m !} \frac{\partial^{m} \widehat{\theta}(\eta ; p)}{\partial p^{m}}\right|_{p=0} .
\end{array}
$$

The value of auxiliary parameter is selected so properly that the series (33) and (34) converge at $p=1$ i.e.

$$
f(\eta)=f_{0}(\eta)+\sum_{m=1}^{\infty} f_{m}(\eta)
$$




$$
\theta(\eta)=\theta_{0}(\eta)+\sum_{m=1}^{\infty} \theta_{m}(\eta)
$$

The general solutions $\left(f_{m}, \theta_{m}\right)$ of Eqs. (23) and (24) in terms of special solutions $\left(f_{m}^{*}, \theta_{m}^{*}\right)$ are given by

$$
\begin{gathered}
f_{m}(\eta)=f_{m}^{*}(\eta)+C 3 * \exp (-\eta)+C 1+C 2 * \eta, \\
\theta_{m}(\eta)=\theta_{m}^{*}(\eta)+c 1 * \exp (-\eta),
\end{gathered}
$$

Skin friction and local Nusselt number can be defined as

$$
\begin{gathered}
C_{f}=\frac{\tau_{w}}{\rho u_{w}^{2}}, \quad N u_{x}=\frac{x q_{w}}{k\left(T_{w}-T_{\infty}\right)}, \\
\tau_{w}=\mu\left(\frac{\partial u}{\partial y}\right)_{y=0}, \quad q_{w}=-k\left(\frac{\partial T}{\partial y}\right)_{y=0} .
\end{gathered}
$$

While the dimensionless forms of skin friction and local Nusselt number are

$$
\frac{1}{\sqrt{2}} C_{f} \operatorname{Re}_{x}^{1 / 2}=f^{\prime \prime}(0), \frac{1}{\sqrt{2}} N u_{x} \operatorname{Re}_{x}^{-1 / 2}=-\theta^{\prime}(0),
$$

where $\operatorname{Re}_{x}=U_{w} x / \nu$.

\section{Convergence of the Homotopy Solutions}

Homotopy analysis method is applied to find the convergence of desired equations which is proposed by Liao [17]. Obviously the series solutions obtained by homotopy analysis method contain the convergence control parameter $\hbar$. This parameter controls the convergence region and the rate of approximation of the HAM solution. To ensure the convergence of the solutions in the admissible range of the values of the auxiliary parameters $\hbar_{f}$ and $\hbar_{\theta}, \hbar$ - curve for 12 -thorder approximations have been made. It is evident from Figs. (2) to (3), that the admissible range of values of $\hbar_{f}$, and $\hbar_{\theta}$ are $-1.4 \leq \hbar_{f} \leq--0.8,-1.2 \leq$, $\hbar_{\theta} \leq-0.3$ The convergence table 1 . is prepared for each of the function up to 16-th order of approximation 


\begin{tabular}{||l||l||l||}
\hline \hline order of convergence & $-\mathrm{f}^{\prime}(0)$ & $-\theta^{\prime}(0)$ \\
\hline \hline 1 & -0.550 & -0.6097 \\
\hline \hline 5 & -0.2686 & -0.5922 \\
\hline \hline 10 & -0.2238 & -0.5894 \\
\hline \hline 15 & -0.2139 & -0.5900 \\
\hline \hline 20 & -0.2109 & -0.5909 \\
\hline \hline 25 & -0.2097 & -0.5916 \\
\hline \hline 30 & -0.2092 & -0.5921 \\
\hline \hline 35 & -0.2091 & -0.5925 \\
\hline \hline 39 & -0.2090 & -0.5924 \\
\hline \hline 40 & -0.2090 & -0.5924 \\
\hline \hline
\end{tabular}

Table 1: Convergence table for velocity and temperature profile

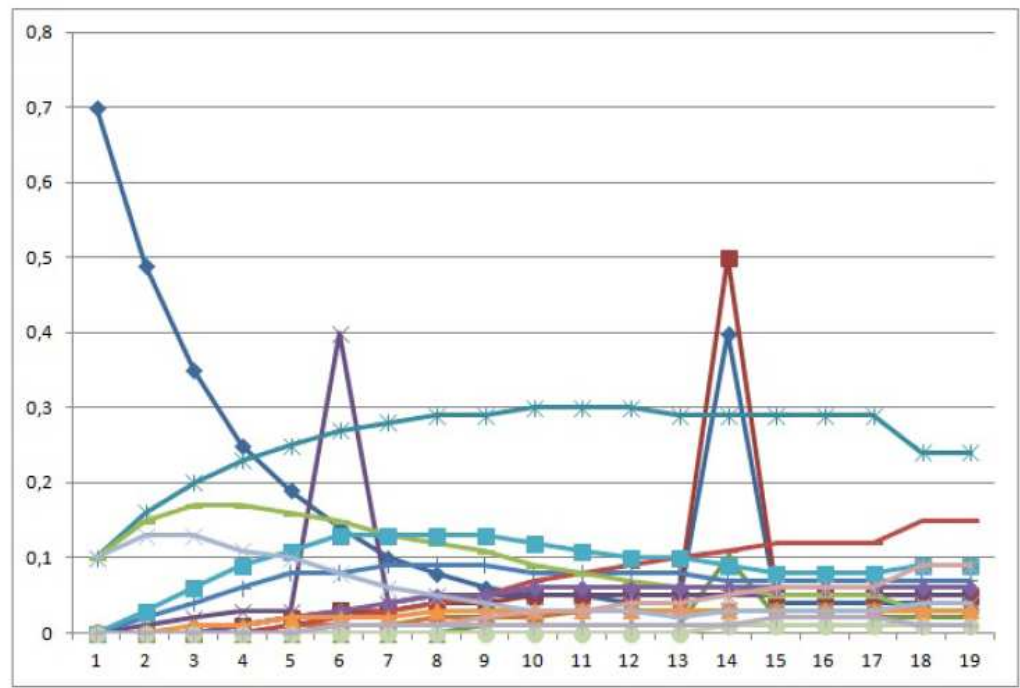

Figure 1: Effect of $R$ on velocity profile

\subsection{Discussion}

Now, we discuss the graphical results of different parameters on velocity and temperature fields. Fig. 1. is plotted for the behavior of Radiation parameter $R$ on velocity field. It is observed that with the increase of radiation parameter $R$ 


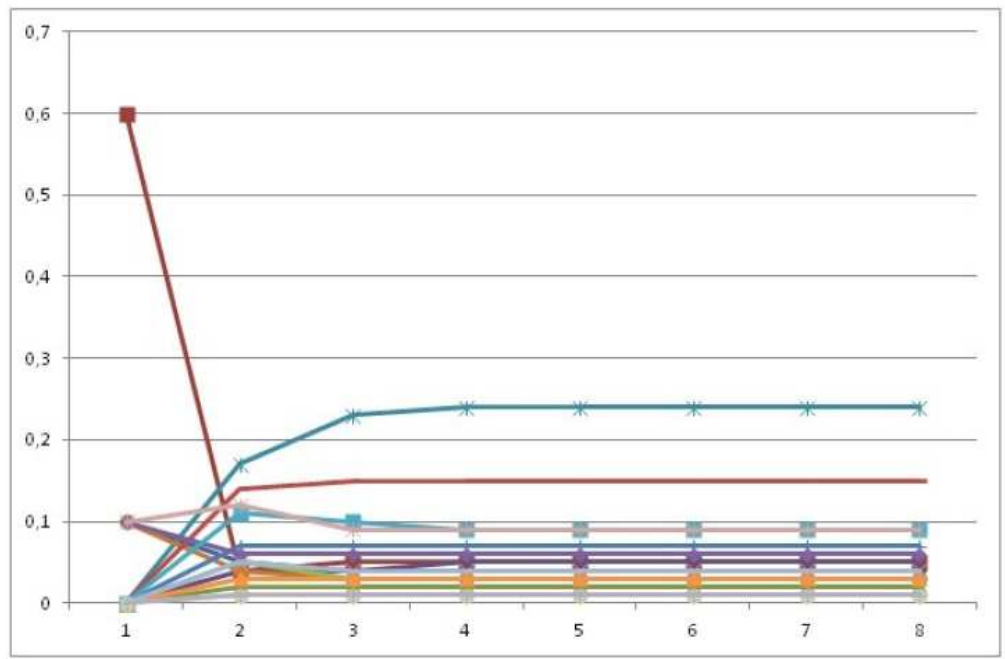

Figure 2: Effect of $\in$ on velocity profile

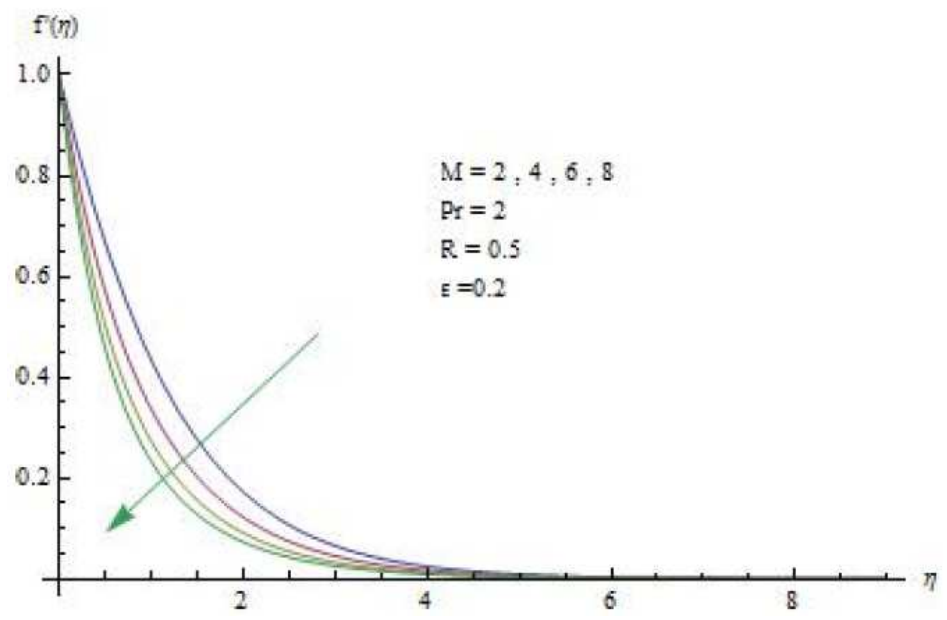

Figure 3: Effect of $M$ on velocity profile

velocity Increase. Fig. 2. is plotted for the behavior of $\in$ on velocity field. It is observed that velocity of the fluid increases with an increase variable thermal conductivity parameter, because due to variable thermal conductivity velocity of particle increases because of variable conduction. The variation of temperature parameter $M$ on velocity profile is sketched in Fig. 3 The characteristics of temperature parameter $M$ on temperature field is shown in Fig. 4 It is ob- 


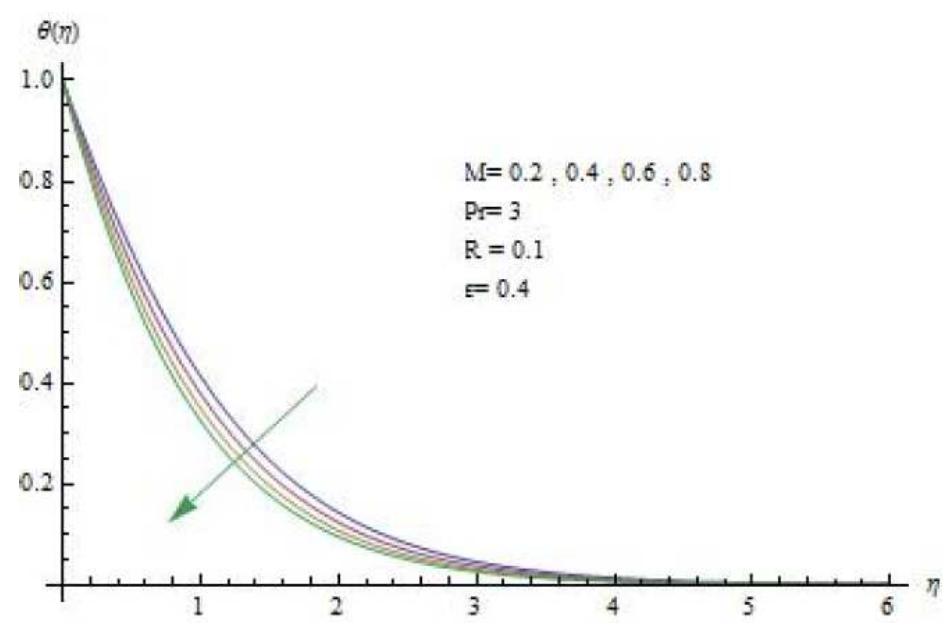

Figure 4: Effect of $M$ on temperature profile

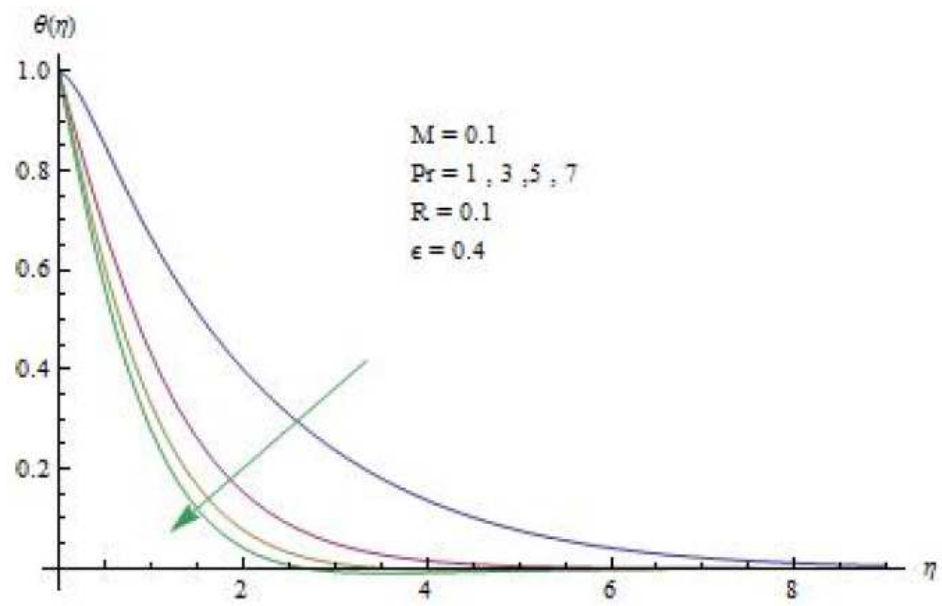

Figure 5: Effect of $\mathrm{Pr}$ on temperature profile

served that temperature profile shows decrease with an increase in temperature parameter, It is due to because $\mathrm{M}$ is inversily proportional to wall temperature which varies linearly from leading edge so by increasing value of $M$ it shows decline behaviour. Fig. 5 is plotted for the effects of Pr on temperature profile, it is seen from figure that by increasing prandtl number viscosity increases as a result temperature decreases. Thermal boundary layer decrease with an increase in Prandtl number. Fig. 6 is plotted for the influence of $\in$ on temperature field. 


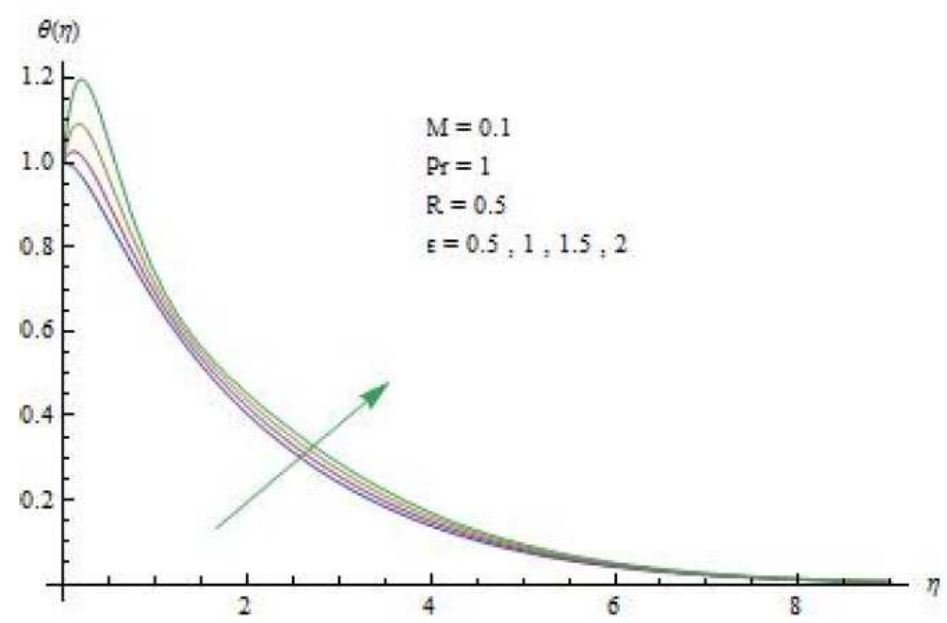

Figure 6: Effect of $\in$ on temperature profile

\begin{tabular}{||l||l||l||l||l||}
\hline \hline $\mathrm{M}$ & $\operatorname{Pr}$ & $\in$ & $\mathrm{R}$ & $\mathrm{f}^{\prime \prime}(0)$ \\
\hline \hline 0.1 & 2 & 1 & 1 & 0.781798 \\
\hline \hline 0.3 & 2 & 1 & 1 & 0.774325 \\
\hline \hline 0.5 & 2 & 1 & 1 & 0.766929 \\
\hline \hline 0.7 & 2 & 1 & 1 & 0.759609 \\
\hline \hline
\end{tabular}

Table 2: The variation of $\frac{1}{2} C_{f} \sqrt{\mathrm{Re}_{x}}$ with respect to $M$

Reading the Table 2, we see that $C_{f} R e_{x}^{-\frac{1}{2}}$. Thus, as Reynold number increases $\left|C_{f}\right|$ decreases. It is well known that as $R e_{x}$ increases the viscous forces start reducing, in turn $\left|C_{f}\right|$ reduces. In the present case, as we increase temperature parameter $\mathrm{M}$ the $\frac{1}{2}\left|C_{f} \mathrm{Re}_{x}\right|$ decreases. As we increase the $\operatorname{Pr}$ $\frac{1}{2}\left|C_{f} \operatorname{Re}_{x}\right|$ decreases. But in the case of variable thermal conductivity parameter the $\frac{1}{2}\left|C_{f} \operatorname{Re}_{x}\right|$ increases.

Reading the Table 3, we see that $N u_{x}^{2} R e_{x}$ so we conclude that as Reynolds number increases, $R e_{x}=\frac{U x}{v}$ increases when viscosity decreases, so decrease in viscosity enhances the magnitude of rate of convectional heat transfer Also, the coefficient of convectional heat depends on Prandtl number $\mathrm{Pr}$, radiation parameter $R$, thermal variable parameter $\epsilon$ and temperature parameter M. The behavior of the coefficient of convectional heat transfer is studied in Table 3. By increasing value of prandtl number value of viscosity increases because it 


\begin{tabular}{||l||l||l||l||l||}
\hline \hline $\mathrm{M}$ & $\mathrm{Pr}$ & $\in$ & $\mathrm{R}$ & $-\theta^{\prime}(0)$ \\
\hline \hline 2 & 1 & 1 & 1 & 0.600000 \\
\hline \hline 2 & 2 & 1 & 1 & 0.630000 \\
\hline \hline 2 & 3 & 1 & 1 & 0.677000 \\
\hline \hline 2 & 1 & 0.1 & 1 & 0.404400 \\
\hline \hline 2 & 1 & 0.2 & 1 & 0.408019 \\
\hline \hline 2 & 1 & 0.3 & 1 & 0.393589 \\
\hline \hline 2 & 1 & 0.4 & 1 & 0.391581 \\
\hline \hline 1 & 1 & 1 & 1 & 0.362597 \\
\hline \hline 2 & 1 & 1 & 1 & 0.377245 \\
\hline \hline 3 & 1 & 1 & 1 & 0.391758 \\
\hline \hline 2 & 1 & 1 & 0.1 & 0.444500 \\
\hline \hline 2 & 1 & 1 & 0.2 & 0.437632 \\
\hline \hline
\end{tabular}

Table 3: Temprature gradient $-\theta^{\prime}(0)$ at the outer surface of plate for different values of $\epsilon, P r$ and $R$

\begin{tabular}{||r||r||r||r||r||}
\hline \hline \multicolumn{4}{||c||}{$M=1$} & \multicolumn{1}{c||}{$\mathrm{M}=2$} \\
\hline \hline$\epsilon$ & HAM Result & FehlburgResult & HAM Result & FehlburgResult \\
\hline \hline 2 & 0.8730 & 0.87301 & 0.78432 & 0.78471 \\
\hline \hline 4 & 0.8834 & 0.88368 & 0.79522 & 0.79511 \\
\hline \hline 6 & 0.8938 & 0.89287 & 0.8043 & 0.80413 \\
\hline \hline 8 & 0.9025 & 0.90093 & 0.81209 & 0.81209 \\
\hline \hline
\end{tabular}

Table 4: The comparison of HAM and Fehlburg method for $f^{\prime \prime}(\eta)$ when $\eta \rightarrow 0$ for variable thermal conductivity $\epsilon$ and temperature parameter M

is ratio b/w momentum diffusitivity and thermal diffusivity there fore Nusselt number increases because Reynolds number decreases.

Table 4 and Table 5 are the caparison of numerical and HAM solution.

\section{Conclusions}

- The main findings of present analysis are listed below 


\begin{tabular}{||r||r||r||r||r||}
\hline \hline \multicolumn{4}{||c||}{ Pr $=1$} & \multicolumn{1}{c||}{ Pr = 2} \\
\hline \hline$R$ & HAM Result & FehlburgResult & HAM Result & FehlburgResult \\
\hline \hline 0.5 & 0.8572 & 0.8514 & 0.7731 & 0.77499 \\
\hline \hline 1 & 0.8730 & 0.87317 & 0.7978 & 0.79994 \\
\hline \hline 1.5 & 0.88971 & 0.88990 & 0.8165 & 0.8198 \\
\hline \hline 2 & 0.9041 & 0.90327 & 0.8303 & 0.83619 \\
\hline \hline
\end{tabular}

Table 5: The comparison of HAM and Fehlburg method for $\theta(\eta)$ when $\eta \rightarrow 0$ for Prandtl number Pr and Radiation parameter $R$

- The behavior of radiation parameter $R$ on velocity and temperature fields are same i.e. both velocity and temperature fields increase with velocity and temperature.

- Temperature field and velocity field decreases for temperature parameter.

- Velocity field increases for values of variable thermal conductivity parameter

\section{References}

[1] L. J. Crane, Flow past a stretching plate, Int.J. Appl. Math, 21(1970)645647.

[2] C. K. Chen, M. I. Char, Heat transfer of a continuous stretching surface with suction or blowing, J. Math. Anal. Appl. 135(1988)568 - 580 .

[3] B.K. Datta, P. Roy, A.S. Gupta, Temperature field in the flow over a stretching sheet with uniform heat flux, Int. Commun. Heat Mass Transfer $12(1985) 89$.

[4] L. Xu, He's homotopy perturbation method for a boundary layer equation in unbounded domain, Comput. Math. Appl. 54(2007)1067 - 1070.

[5] B. Raftari, A. Yıldırım, The application of homotopy perturbation method for MHD flows of UCM fluids above porous stretching sheets, Comput. Math. Appl. 59(2010)3328 - 3337.

[6] P.D. Ariel, The three-dimensional flow past a stretching sheet and the homotopy perturbation method, Comput. Math. Appl. 54(2007)920 - 925. 
[7] P.D. Ariel, Extended homotopy perturbation method and computation of flow past a stretching sheet, Comput. Math. Appl. 58(2009)2402 - 2409.

[8] J. Gary, D. R. Kassoy, H. Tadjeran, A. Zebib, The effects of significant viscosity variation on convective heat transport in water saturated porous medium, J. Fluid Mech. 117(1982)233 - 249.

[9] S. Mukhopadhyay, G.C. Layek, S.A. Samad, Study of MHD boundary layer flow over a heated stretching sheet with variable conductivity, Int. J. Heat Mass Transfer 48(2005)4460 - 4466.

[10] T.R. Mahapatra, A.G. Gupta, Heat transfer in stagnation point flow towards a stretching sheet, Int. J. Heat and Mass Transfer 38(2002)517-521.

[11] A.J. Chamkha, Non-Darcy Hydromagnetic Free Convection in Porous Media, Int. Commun. Heat Mass Transfer 23(1996)875 - 887.

[12] K. Vajravelu, A. Hadjinicolaou, Convective Heat Transfer in an Electrically Conducting Fluid at a Stretching Surface with Uniform Free Stream, Int. J. Engng Sci. 35(1997)1237 - 1244.

[13] S.J. Liao, Introduction to the Homotopy Analysis Method, Chapman \& Hall/CRC Press, Boca Raton, 2003.

[14] S. Mukhopadhayay, G.C.Layek, S.A.Samad, Study of MHD boundary layer flow over a heated stretching sheet with variable conductivity, Int. J. Heat Mass transfer 48(2005)4460 - 44.

[15] A. Ishak, R. Nazar and I. Pop,Boundary layer flow with variable thermal conductivity, Nonlinear Analysis, Real World Application. 10, 2909(2009).

[16] M. A. Seddeek, F. A. Salama, The effects of temperature dependent viscosity and thermal conductivity on unsteady MHD convective heat transfer past a semi-infinite vertical porous moving plate with variable suction, Int. Comp. Mater, 40(2007)186 - 192.

[17] T. C. Chiam, Heat transfer in a fluid with variable thermal conductivity over a linearly stretching sheet, Int. J. Acta Mech, 129(1998)63 - 72. 